\title{
Modified viscosity type iteration for total asymptotically nonexpansive mappings in CAT(0) spaces and its application to optimization problems
}

\author{
Wiyada Kumam ${ }^{\mathrm{a}, *}$, Nuttapol Pakkaranang ${ }^{\mathrm{b}}$, Poom Kumam ${ }^{\mathrm{b}, \mathrm{c}, \mathrm{d}, *}$ \\ a Program in Applied Statistics, Department of Mathematics and Computer Science, Faculty of Science and Technology, Rajamangala \\ University of Technology Thanyaburi, Thanyaburi, Pathumthani 12110, Thailand. \\ ${ }^{b}$ KMUTTFixed Point Research Laboratory, Department of Mathematics, Room SCL 802 Fixed Point Laboratory, Science Laboratory \\ Building, Faculty of Science, King Mongkut's University of Technology Thonburi (KMUTT), 126 Pracha-Uthit Road, Bang Mod, Thung \\ Khru, Bangkok 10140, Thailand. \\ ${ }^{c}$ KMUTT-Fixed Point Theory and Applications Research Group, Theoretical and Computational Science Center (TaCS), Science \\ Laboratory Building, Facuty of Science, King Mongkut's University of Technology Thonburi (KMUTT), 126 Pracha-Uthit Road, Bang \\ Mod, Thung Khru, Bangkok 10140, Thailand. \\ ${ }^{d}$ Department of Medical Research, China Medical University Hospital, China Medical University, Taichung 40402, Taiwan.
}

\begin{abstract}
In this paper, we introduce a modified two-step viscosity iteration process for total asymptotically nonexpansive mappings in CAT(0) spaces. We prove strong convergence of the proposed iteration process to a fixed point of total asymptotically nonexpansive mappings in CAT(0) spaces, which also shows that the limit of the sequence generated by proposed iteration process solves the solution of the variational inequality. We also provide illustrating a numerical example for supporting our main results. Moreover, we show the existence of solutions of our consequently results for some applications.
\end{abstract}

Keywords: Viscosity approximation methods, total asymptotically nonexpansive mapping, variational inequality, CAT(0) spaces.

2010 MSC: 47H09, 47J20, 47J25.

(C)2018 All rights reserved.

\section{Introduction}

Let $\mathrm{C}$ be a nonempty closed subset of a metric space $\mathrm{X}$. Let $\mathrm{T}: \mathrm{C} \rightarrow \mathrm{C}$ be a self-mapping. Recall that a mapping $\mathrm{T}$ is said to be:

(i) contraction if there exist a constant $k \in[0,1)$ such that $d(T x, T y) \leqslant k d(x, y), \quad \forall x, y \in C$;

(ii) nonexpansive if $d(T x, T y) \leqslant d(x, y), \forall x, y \in C$;

\footnotetext{
*Corresponding authors

Email addresses: wiyada.kum@rmutt.ac.th (Wiyada Kumam), nuttapol.pak@mail.kmutt.ac.th (Nuttapol Pakkaranang), poom.kum@kmutt.ac.th (Poom Kumam)
}

doi: $10.22436 /$ jnsa.011.02.10

Received: 2017-10-22 Revised: 2017-11-26 Accepted: 2017-12-01 
(iii) asymptotically nonexpansive if there exists a sequence $\left\{k_{n}\right\}$ in $[1, \infty)$ with $\lim _{n \rightarrow \infty} k_{n}=1$ such that $d\left(T^{n} x, T^{n} y\right) \leqslant k_{n} d(x, y), \forall x, y \in C$ and $\forall n \geqslant 1 ;$

(iv) uniformly L-Lipschitz if there exist a constant $L>0$ such that $d\left(T^{n} x, T^{n} y\right) \leqslant L d(x, y), \forall x, y \in C$ and $\forall \mathrm{n} \geqslant 1$.

In 2006, Alber et al. [4] first introduced the concept of the class of total asymptotically nonexpansive mappings. Recall that the self mapping $T$ is said to be $(\mu, \xi, \psi)$-total asymptotically nonexpansive, if there exist non-negative real sequences $\left\{\mu_{n}\right\},\left\{\xi_{n}\right\}$ with $\mu_{n} \rightarrow 0, \xi_{n} \rightarrow 0$ as $n \rightarrow \infty$ and a continuous strictly increasing function $\psi:[0, \infty) \rightarrow[0, \infty)$ with $\psi(0)=0$ such that

$$
d\left(T^{n} x, T^{n} y\right) \leqslant d(x, y)+\mu_{n} \psi(d(x, y))+\xi_{n}, \forall n \geqslant 1, x, y \in C
$$

From definition, it is easy to know that each nonexpansive mapping be an asymptotically nonexpansive with $k_{n}:=1$, and each asymptotically nonexpansive mapping be a $(\mu, \xi, \psi)$-total asymptotically nonexpansive mapping with $\mu_{n}:=k_{n}-1, \xi_{n}:=0, \forall n \geqslant 1$ and $\psi(t)=t, t \geqslant 0$.

A point $x \in C$ is called a fixed point of $T$ if $x=T(x)$. We denote with $F(T)$ the fixed point set of $T$. A sequence $\left\{x_{n}\right\}$ in $C$ is said to be approximating fixed point sequence (in short term, AFPS) of T if

$$
\lim _{n \rightarrow \infty} d\left(x_{n}, T x_{n}\right)=0 .
$$

The class of such a mapping generalized the basic concepts of asymptotically nonexpansive mappings introduced by Kirk and Goebel [17]. Likewise, the basics concept of nearly asymptotically nonexpansive mappings introduced by Sahu [25].

Recall that $(X, d)$ be a metric space. Such a space is called a CAT(0) space if it is geodesically connected and every geodesic triangle in $X$ is at least as 'thin' as its comparison triangle in Euclidean plane $\mathbb{R}^{2}$. Some examples of $\mathrm{CAT}(0)$ spaces are

(C1) R-trees;

(C2) simply connected Riemannian manifold of non-positive sectional curvature;

(C3) Hilbert spaces [7];

(C4) Euclidean spaces [8].

A subset $K$ of a $\operatorname{CAT}(0)$ space $X$ is said to be convex if, for any $x, y \in K,[x, y] \subset K$, where $[x, y]:=$ $\{\lambda x \oplus(1-\lambda) y ; 0 \leqslant \lambda \leqslant 1\}$ is the unique geodesic joining $x$ and $y$. For although discussion of $\mathrm{CAT}(0)$ spaces, some fundamental geometric properties and important conclusions, we refer to Bridson, Haefliger [7] and some authors $[3,8,11,12,21-24,29,30]$. A complete CAT(0) space is often called a Hadamard space.

In 2012, Chang et al. [10] studied and proved demiclosedness principle and some convergence theorems for total asymptotically nonexpansive mappings in a CAT(0) space. Since then, some convergence theorems of many iterative algorithms for a type of such a mapping has been rapidly developed and several of articles have cited in references (see e.g.[5, 9, 18, 28, 31, 32, 37-39]).

In 2008 Berg and Nikolaev [6] introduced the concept of quasi-linearization in X.

Denote a pair $(p, q) \in X \times X$ by $\overrightarrow{p q}$ and call it a vector. Then quasi-linearization is defined as a mapping $\langle\cdot, \cdot\rangle:(\mathrm{X} \times \mathrm{X}) \times(\mathrm{X} \times \mathrm{X}) \rightarrow \mathbb{R}$ such that

$$
\langle\overrightarrow{p q}, \overrightarrow{r s}\rangle=\frac{1}{2}\left(d^{2}(p, s)+d^{2}(q, r)-d^{2}(p, r)-d^{2}(q, s)\right)
$$

for all $p, q, r, s \in X$.

In 2012, Dehghan and Rooin [13] introduced the basic of duality mapping in a CAT(0) space and studied its relation with subdifferential by using the concept of quasi-linearization. Then they proposed a characterization of metric projection in a CAT(0) space as follows. 
Let $(X, d)$ be a complete CAT( 0$)$ space and $C$ a nonempty closed convex subset of $X$. Let $u \in C$ and $x \in$ $X$. Then $u=P_{C} x$ if and only if

$$
\langle\overrightarrow{y u}, \overrightarrow{u x}\rangle \geqslant 0, \quad \forall y \in C .
$$

In 2013, Wangkeeree and Preechasilp [34] used the concept of quasi-linearization and studied strong convergence theorems of viscosity iteration for nonexpansive mapping in a complete CAT(0) space as follows.

Let $f$ is contraction on $C$ such that for arbitrary chosen $x_{1} \in C$, the sequence $\left\{x_{n}\right\}$ is generated by

$$
x_{n+1}=\alpha_{n} f\left(x_{n}\right) \oplus\left(1-\alpha_{n}\right) T x_{n}, \forall n \geqslant 1,
$$

where $\left\{\alpha_{n}\right\} \subset[0,1]$. They proved the sequence defined by (1.1) converging strongly to $\tilde{p}$ such that $\tilde{p}=$ $P_{F(T)} f(\tilde{p})$ which is the unique solution of the variational inequality:

$$
\langle\overrightarrow{\tilde{p} f(\tilde{p})}, \overrightarrow{p \vec{p}}\rangle \geqslant 0, \quad p \in F(T) .
$$

In 2015, Wangkeeree et al. [33] studied the strong convergence theorem of the viscosity iteration for asymptotically nonexpansive mapping in a complete CAT(0) space: Let $f$ is contraction on $\mathrm{C}$ such that the sequence $\left\{x_{n}\right\}$ for arbitrary chosen

$$
\left\{\begin{array}{l}
x_{1} \in C \\
x_{n+1}=\alpha_{n} f\left(x_{n}\right) \oplus\left(1-\alpha_{n}\right) T^{n} x_{n}, \forall n \geqslant 1
\end{array}\right.
$$

Where $\left\{\alpha_{n}\right\} \subset[0,1]$. They proved that the sequence defined by (1.3) converges strongly to $\tilde{p}=P_{F(T)} f(\tilde{p})$ which is the unique solution of the variational inequality (1.2).

Motivated and inspired by (1.3) in [33], we introduce the modified two-step viscosity iteration process for a total asymptotically nonexpansive mapping in a complete CAT(0) space, for arbitrary chosen $x_{1} \in \mathrm{C}$ the sequence generated by

$$
\left\{\begin{array}{l}
x_{n+1}=\alpha_{n} f\left(x_{n}\right) \oplus\left(1-\alpha_{n}\right) T^{n} y_{n} \\
y_{n}=\beta_{n} x_{n} \oplus\left(1-\beta_{n}\right) T^{n} x_{n}
\end{array}\right.
$$

for all $n \geqslant 1$, where $\alpha_{n}, \beta_{n} \in[0,1]$. $T: C \rightarrow C$ is a total asymptotically nonexpansive mapping and $f: C \rightarrow C$ is a contraction mapping.

The purpose of this paper is to prove strong convergence theorem of iterative scheme generated by (1.4) for a total asymptotically nonexpansive mapping in a CAT(0) space under some conditions. We also show that the limit of the sequence generated by (1.4) solves the solution of the variational inequality (1.2). We then give a numerical example for supporting our main results. Moreover, we show the existence of solutions of our consequently results for some applications.

\section{Preliminaries}

In this section, we always suppose that $X$ is a $C A T(0)$ space and write $(1-t) x \oplus$ ty for the unique point $w$ in the geodesic segment joining form $x$ to $y$, which is $[x, y]=(1-\lambda) x \oplus \lambda y: \lambda \in[0,1]$,

$$
d(w, x)=\lambda d(x, y) \text { and } d(w, y)=(1-\lambda) d(x, y) .
$$

Lemma 2.1 ([16]). Let $\mathrm{K}$ be a $C A T(0)$ space. For all $x, y, z \in X$ and $\lambda, \gamma \in[0,1]$, we have the following:

(i) $d(\lambda x \oplus(1-\lambda) y, z) \leqslant \lambda d(x, z)+(1-\lambda) d(y, z)$;

(ii) $d^{2}(\lambda x \oplus(1-\lambda) y, z) \leqslant \lambda d^{2}(x, z)+(1-\lambda) d^{2}(y, z)-\lambda(1-\lambda) d^{2}(x, y)$;

(iii) $\mathrm{d}(\lambda x \oplus(1-\lambda) \mathrm{y}, \gamma x \oplus(1-\gamma) \mathrm{y})=|\lambda-\gamma| \mathrm{d}(x, y)$;

(iv) $\mathrm{d}(\lambda x \oplus(1-\lambda) \mathrm{y}, \mathrm{tu} \oplus(1-\lambda) w) \leqslant \lambda d(x, u)+(1-\lambda) d(y, w)$. 
Next, we refer to some elementary properties about CAT(0) spaces as follows.

Let $\left\{x_{n}\right\}$ be a bounded sequence in a $\operatorname{CAT}(0)$ space $(X, d)$. For $x \in X$, we set

$$
r\left(x,\left\{x_{n}\right\}\right)=\limsup _{n \rightarrow \infty} d\left(x, x_{n}\right) .
$$

The asymptotic radius $r\left(\left\{x_{n}\right\}\right)$ of $\left\{x_{n}\right\}$ is given by

$$
r\left(\left\{x_{n}\right\}\right)=\inf \left\{r\left(x,\left\{x_{n}\right\}\right): x \in X\right\},
$$

and the asymptotic center $A\left(\left\{x_{n}\right\}\right)$ of $\left\{x_{n}\right\}$ is the set

$$
A\left(\left\{x_{n}\right\}\right)=\left\{x \in X: r\left(x,\left\{x_{n}\right\}\right)=r\left(\left\{x_{n}\right\}\right)\right\} .
$$

It is well known that in a complete CAT( 0$)$ space, $A\left(\left\{x_{n}\right\}\right)$ consists of exactly one point [15].

In 1976, the concept of convergence in a general metric space introduced by Lim [20] setting which is called ' $\Delta$-convergent'. Later, Kirk and Panyanak [19] used the concept of $\Delta$-convergent to prove in a $\mathrm{CAT}(0)$ space.

Definition 2.2 ([26]). A sequence $\left\{x_{n}\right\}$ in $X$ is said to $\Delta$-converge to $x \in X$ if $x$ is the unique asymptotic center of $\left\{u_{n}\right\}$ for every subsequence $\left\{u_{n}\right\}$ of $\left\{x_{n}\right\}$. In this case we write $\Delta-\lim _{n \rightarrow \infty} x_{n}=x$ and call $x$ the $\Delta$-limit of $\left\{x_{n}\right\}$.

Kakavandi and Amini [2] introduced the following notion of quasi-linearization of convergence.

A sequence $\left\{x_{n}\right\}$ in the complete $\operatorname{CAT}(0)$ space $(X, d) w$-convergences to $x \in X$ if

$$
\lim _{n \rightarrow \infty}\left\langle\overrightarrow{x x_{n}}, \overrightarrow{x y}\right\rangle=0,
$$

i.e. $\lim _{n \rightarrow \infty}\left(d^{2}\left(x_{n}, x\right)-d^{2}\left(x_{n}, y\right)+d^{2}(x, y)\right)=0, \forall y \in X$.

It is obvious that convergence in the metric implies $w$-convergence, and it is easy to investigate that $w$-convergence implies $\Delta$-convergence [1], but it is showed in [2] that the inverse is false. However, the following lemma shows another characterization of $\Delta$-convergence as well as, more explicitly, a relation between $w$-convergence and $\Delta$-convergence.

Lemma 2.3 ([19]). Every bounded sequence in a complete CAT(0) space always has a $\Delta$-convergent subsequence.

Lemma 2.4 ([14]). If $\mathrm{C}$ is a closed convex subset of a complete $C A T(0)$ space and $\left\{x_{n}\right\}$ is a bounded sequence in $C$, then the asymptotic center of $\left\{x_{n}\right\}$ is in $\mathrm{C}$.

Lemma 2.5 ([18]). If $\mathrm{C}$ is a closed convex subset of $\mathrm{X}$ and $\mathrm{T}: \mathrm{C} \rightarrow \mathrm{X}$ is a total asymptotically nonexpansive mapping, then the conditions $\left\{x_{n}\right\} \Delta$-convergence to $\mathrm{x}$ and $\mathrm{d}\left(\mathrm{x}_{\mathrm{n}}, \mathrm{T} \mathrm{x}_{\mathrm{n}}\right) \rightarrow 0$, and imply $\mathrm{x} \in \mathrm{C}$ and $\mathrm{x} \in \mathrm{F}(\mathrm{T})$.

Lemma 2.6 ([1]). Let $X$ be a complete $C A T(0)$ space, $\left\{x_{n}\right\}$ be a sequence in $X$ and $x \in X$. Then $\left\{x_{n}\right\} \Delta$-converges to $x$ if and only if $\limsup _{n \rightarrow \infty}\left\langle\overrightarrow{x x_{n}}, \overrightarrow{x y}\right\rangle \leqslant 0$ for all $y \in X$.

The following two important lemmas can be found in [34].

Lemma 2.7 ([34]). Let $\mathrm{X}$ be a complete $C A T(0)$ space. Then for all $\mathrm{u}, \mathrm{x}, \mathrm{y} \in \mathrm{X}$, the following inequality holds

$$
d^{2}(x, u) \leqslant d^{2}(y, u)+2\langle\overrightarrow{x y}, \overrightarrow{x u}\rangle
$$

Lemma 2.8 ([34]). Let $\mathrm{X}$ be a $C A T(0)$ space. For any $\mathrm{t} \in[0,1]$ and $\mathrm{u}, \boldsymbol{v} \in \mathrm{X}$, let $\mathrm{u}_{\mathrm{t}}=\mathrm{t} \mathrm{u} \oplus(1-\mathrm{t}) \boldsymbol{v}$. Then, for all $x, y \in X$,

(i) $\left\langle\overrightarrow{u_{t} x}, \overrightarrow{u_{t} \vec{y}}\right\rangle \leqslant t\left\langle\overrightarrow{u x}, \overrightarrow{u_{t} \vec{y}}\right\rangle+(1-t)\left(\overrightarrow{v x}, \overrightarrow{u_{t} y}\right\rangle$;

(ii) $\left\langle\overrightarrow{u_{t}} \vec{x}, \overrightarrow{u y}\right\rangle \leqslant t\langle\overrightarrow{u x}, \overrightarrow{u y}\rangle+(1-t)\langle\overrightarrow{v x}, \overrightarrow{u y}\rangle$ and $\left\langle\overrightarrow{u_{t} x}, \overrightarrow{u y}\right\rangle \leqslant t\langle\overrightarrow{u x}, \overrightarrow{v y}\rangle+(1-t)(\overrightarrow{v x}, \overrightarrow{v y}\rangle$. 
Lemma 2.9 ([35]). Let $\left\{a_{n}\right\}$ be a sequence of non-negative real numbers satisfying the property

$$
a_{n+1} \leqslant\left(1-\alpha_{n}\right) a_{n}+\alpha_{n} \beta_{n}, \quad n \geqslant 0,
$$

where $\left\{\alpha_{n}\right\} \subseteq(0,1)$ and $\left\{\beta_{n}\right\} \subseteq \mathbb{R}$ such that

(i) $\sum_{n=0}^{\infty} \alpha_{n}=\infty$;

(ii) $\limsup _{n \rightarrow \infty} \beta_{n} \leqslant 0$ or $\sum_{n=0}^{\infty}\left|\alpha_{n} \beta_{n}\right|<\infty$.

Then $\left\{\mathrm{a}_{\mathrm{n}}\right\}$ converges to zero, as $\mathrm{n} \rightarrow \infty$.

\section{Main results}

\subsection{Strong convergence theorem}

Theorem 3.1. Let $C$ be nonempty closed and convex subset of a complete $C A T(0)$ space $\mathrm{X}$ and $\mathrm{T}: \mathrm{C} \rightarrow \mathrm{C}$ be a total asymptotically nonexpansive mapping with sequence $\mu_{n}$ and $\xi_{n}$ satisfying $\sum_{n=1}^{\infty} \mu_{n}<\infty$ and $\sum_{n=1}^{\infty} \xi_{n}<\infty$ such that $\mathrm{F}(\mathrm{T}) \neq \emptyset$. Let $\mathrm{f}$ be a contraction on $\mathrm{C}$ with the coefficient $\mathrm{k} \in[0,1)$ and for arbitrary initial point $\mathrm{x}_{1} \in \mathrm{C}$. Let sequence $\left\{x_{n}\right\}$ be generated by (1.4) satisfying the following conditions:

(i) there exist constant $M>0$ such that $\psi(r) \leqslant M r, r \geqslant 0$;

(ii) $\lim _{n \rightarrow \infty} \alpha_{n}=0$;

(iii) $\sum_{n=1}^{\infty} \alpha_{n}=\infty$;

(iv) T satisfies the asymptotically regular $\lim _{n \rightarrow \infty} \mathrm{d}\left(\mathrm{x}_{\mathrm{n}}, \mathrm{T}^{\mathrm{n}} \mathrm{x}_{\mathrm{n}}\right)=0$.

Then the sequence $\left\{x_{n}\right\}$ converges strongly to $\tilde{p}$ which is a fixed point of $T$ such that $\tilde{p} \in \mathrm{P}_{\mathrm{F}(\mathrm{T})} \mathrm{f}(\tilde{\mathrm{p}})$ and also equivalent to the solution of the variational inequality (1.2).

Proof.

Step I. First, for all $w \in C$ we define a mapping $F_{w}: C \rightarrow C$ such that

$$
F_{w}(x)=\alpha f(w) \oplus(1-\alpha) T^{n}\left(\beta \oplus(1-\beta) T^{n} x\right)
$$

for all $n \geqslant 1$, where $\alpha, \beta \in(0,1)$ and we will show that $F_{w}$ is a contraction mapping.

Indeed, it follows from Lemma 2.1 that, for all $x, y \in C$

$$
\begin{aligned}
& \mathrm{d}\left(\mathrm{F}_{w}(\mathrm{x}), \mathrm{F}_{w}(\mathrm{y})\right)=\mathrm{d}\left(\alpha \mathrm{f}(w) \oplus(1-\alpha) \mathrm{T}^{\mathrm{n}}\left(\beta x \oplus(1-\beta) \mathrm{T}^{\mathrm{n}} \mathrm{x}\right),\left(\alpha \mathrm{f}(w) \oplus(1-\alpha) \mathrm{T}^{\mathrm{n}}\left(\beta \mathrm{y} \oplus(1-\beta) \mathrm{T}^{\mathrm{n}} \mathrm{y}\right)\right)\right. \\
& \leqslant \alpha d(f(w), f(w))+(1-\alpha) d\left(T^{n}\left(\beta x \oplus(1-\beta) T^{n} x\right), T^{n}\left(\beta y \oplus(1-\beta) T^{n} y\right)\right) \\
& \leqslant(1-\alpha)\left\{d\left(\beta x \oplus(1-\beta) T^{n} x, \beta y \oplus(1-\beta) T^{n} y\right)\right. \\
& +\mu_{n} \psi\left(d\left(\beta x \oplus(1-\beta) T^{n} x, \beta y \oplus(1-\beta) T^{n} y\right)+\xi_{n}\right\} \\
& \leqslant(1-\alpha)\left\{\beta d(x, y)+(1-\beta) d\left(T^{n} x, T^{n} y\right)+\mu_{n} \psi(\beta d(x, y)\right. \\
& \left.\left.+(1-\beta) d\left(T^{n} x, T^{n} y\right)\right)+\xi_{n}\right\} \\
& \leqslant(1-\alpha)\left\{\beta d(x, y)+(1-\beta)\left(d(x, y)+\mu_{n} \psi(d(x, y))+\xi_{n}\right)+\mu_{n} \psi(\beta d(x, y)\right. \\
& \left.+(1-\beta)\left(d(x, y)+\mu_{n} \psi(d(x, y))+\xi_{n}\right)+\xi_{n}\right\} \\
& \leqslant(1-\alpha)\left\{\beta d(x, y)+(1-\beta)\left(\left(1+\mu_{n} M\right) d(x, y)+\xi_{n}\right)+\mu_{n} \psi(\beta d(x, y)\right. \\
& \left.+(1-\beta)\left(\left(1+\mu_{n} M\right) d(x, y)+\xi_{n}\right)+\xi_{n}\right\} \\
& =(1-\alpha)\left\{d(x, y)+(1-\beta)\left(\mu_{n} M d(x, y)+\xi_{n}\right)+\mu_{n} \psi(d(x, y)\right. \\
& \left.\left.+(1-\beta)\left(\mu_{n} \operatorname{Md}(x, y)+\xi_{n}\right)\right)+\xi_{n}\right\} \\
& \leqslant(1-\alpha) \mathrm{d}(x, y) \text {. }
\end{aligned}
$$

Since $\sum_{n=1}^{\infty} \mu_{n}<\infty$ and $\sum_{n=1}^{\infty} \xi_{n}<\infty$. This implies that $F_{w}$ is a contraction mapping. That means sequence $\left\{x_{n}\right\}$ is well define. 
Step II. Next, we prove that $\left\{x_{n}\right\}$ is bounded.

Let $p \in F(T)$ to deduce that

$$
\begin{aligned}
d\left(y_{n}, p\right) & =d\left(\beta_{n} x_{n} \oplus\left(1-\beta_{n}\right) T^{n} x_{n}, p\right) \\
& \leqslant \beta_{n} d\left(x_{n}, p\right)+\left(1-\beta_{n}\right) d\left(T^{n} x_{n}, p\right) \\
& \leqslant \beta_{n} d\left(x_{n}, p\right)+\left(1-\beta_{n}\right)\left(d\left(x_{n}, p\right)+\mu_{n} \psi\left(d\left(x_{n}, p\right)\right)+\xi_{n}\right) \\
& \leqslant\left(1+\left(1-\beta_{n}\right) \mu_{n} M\right) d\left(x_{n}, p\right)+\left(1-\beta_{n}\right) \xi_{n}
\end{aligned}
$$

and we have

$$
\begin{aligned}
d\left(x_{n+1}, p\right)= & d\left(\alpha_{n} f\left(x_{n}\right) \oplus\left(1-\alpha_{n}\right) T^{n} y_{n}, p\right) \\
\leqslant & \alpha_{n} d\left(f\left(x_{n}\right), p\right)+\left(1-\alpha_{n}\right) d\left(T^{n} y_{n}, p\right) \\
\leqslant & \alpha_{n}\left(d\left(f\left(x_{n}\right), f(p)\right)+d(f(p), p)\right)+\left(1-\alpha_{n}\right)\left(d\left(y_{n}, p\right)+\mu_{n} \psi\left(d\left(y_{n}, p\right)\right)+\xi_{n}\right) \\
\leqslant & \alpha_{n} k d\left(x_{n}, p\right)+\alpha_{n} d(f(p), p)+\left(1-\alpha_{n}\right)\left(\left(1+\mu_{n} M\right) d\left(y_{n}, p\right)+\xi_{n}\right) \\
\leqslant & \alpha_{n} k d\left(x_{n}, p\right)+\alpha_{n} d(f(p), p) \\
& +\left(1-\alpha_{n}\right)\left(\left(1+\mu_{n} M\right)\left(\left(1+\left(1-\beta_{n}\right) \mu_{n} M\right) d\left(x_{n}, p\right)+\left(1-\beta_{n}\right) \xi_{n}\right)+\xi_{n}\right. \\
= & \left(\alpha_{n} k+\left(1-\alpha_{n}\right)\left(1+\mu_{n} M\right)\left(1+\left(1-\beta_{n}\right) \mu_{n} M\right) d\left(x_{n}, p\right)\right. \\
& +\left(\left(1-\alpha_{n}\right)\left(1-\beta_{n}\right)+1\right) \xi_{n}+\alpha_{n} d(f(p), p) .
\end{aligned}
$$

Since $\sum_{n=1}^{\infty} \mu_{n}<\infty$ and $\sum_{n=1}^{\infty} \xi_{n}<\infty$, we obtain

$$
d\left(x_{n+1}, p\right) \leqslant\left(1-(1-k) \alpha_{n}\right) d\left(x_{n}, p\right)+\alpha_{n} d(f(p), p) \leqslant \max \left\{d\left(x_{n}, p\right), \frac{1}{1-k} d(f(p), p)\right\}
$$

for all $n \geqslant 0$. This implies that $\left\{x_{n}\right\}$ is bounded, and so $\left\{f\left(x_{n}\right)\right\},\left\{T^{n} x_{n}\right\}$, and $\left\{T^{n} y_{n}\right\}$ are also bounded.

Next, we claim that sequence $\left\{x_{n}\right\}$ is AFPS. That means $\lim _{n \rightarrow \infty} d\left(x_{n}, T x_{n}\right)=0$. Indeed, we have

$$
\begin{aligned}
d\left(x_{n+1}, x_{n}\right) & \leqslant d\left(x_{n+1}, T^{n} x_{n}\right)+d\left(T^{n} x_{n}, x_{n}\right) \\
& =d\left(\alpha_{n} f\left(x_{n}\right) \oplus\left(1-\alpha_{n}\right) T^{n} y_{n}, T^{n} x_{n}\right)+d\left(T^{n} x_{n}, x_{n}\right) \\
& \leqslant \alpha_{n} d\left(f\left(x_{n}\right), T^{n} x_{n}\right)+\left(1-\alpha_{n}\right) d\left(T^{n} y_{n}, T^{n} x_{n}\right)+d\left(T^{n} x_{n}, x_{n}\right) \\
& \rightarrow 0 \text { as } n \rightarrow \infty, \\
d\left(x_{n}, T^{n-1} x_{n}\right) & =d\left(\alpha_{n-1} f\left(x_{n-1}\right) \oplus\left(1-\alpha_{n-1}\right) T^{n-1} x_{n-1}, T^{n-1} x_{n}\right) \\
& \leqslant \alpha_{n-1} d\left(f\left(x_{n-1}\right), T^{n-1} x_{n}\right)+\left(1-\alpha_{n-1}\right) d\left(T^{n-1} x_{n-1}, T^{n-1} x_{n}\right) \\
& \leqslant \alpha_{n-1} d\left(f\left(x_{n-1}\right), T^{n-1} x_{n}\right)+\left(1-\alpha_{n-1}\right)\left(1+\mu_{n} M\right) d\left(x_{n+1}, x_{n}\right)+\xi_{n} \\
& \rightarrow 0 \text { as } n \rightarrow \infty .
\end{aligned}
$$

Therefore

$$
\begin{aligned}
d\left(x_{n}, T x_{n}\right) & \leqslant d\left(x_{n}, T^{n} x_{n}\right)+d\left(T^{n} x_{n}, T x_{n}\right) \\
& =d\left(x_{n}, T^{n} x_{n}\right)+d\left(T T^{n-1} x_{n}, T x_{n}\right) \\
& \leqslant d\left(x_{n}, T^{n} x_{n}\right)+\operatorname{Ld}\left(T^{n-1} x_{n}, x_{n}\right) \\
& \rightarrow 0 \text { as } n \rightarrow \infty .
\end{aligned}
$$

Since $\left\{x_{n}\right\}$ is bounded, there exists a subsequence $\left\{x_{n_{j}}\right\}$ of $\left\{x_{n}\right\}$, which $\Delta$-converges to $\tilde{p}$ such that $\tilde{p}=P_{F(T)} f(\tilde{p})$, which is also equivalent to the following solution of the variational inequality (1.2).

By Lemmas 2.3 and 2.5 we can suppose that $\left\{x_{n_{j}}\right\} \Delta$-converges to a point $\tilde{p}$ and $\tilde{p} \in F(T)$. It follows from Lemma 2.8 (i) that

$$
d^{2}\left(x_{n_{j}}, \tilde{p}\right)=\left\langle\overrightarrow{x_{n_{j}} \tilde{p}}, \overrightarrow{x_{n_{j}} \tilde{p}}\right\rangle \leqslant \alpha_{n_{j}}\left\langle\overrightarrow{f\left(x_{n_{j}}\right) \tilde{p}}, \overrightarrow{x_{n_{j}} \tilde{p}}\right\rangle+\left(1-\alpha_{n_{j}}\right)\left\langle\overrightarrow{T^{n_{j}} x_{n_{j}} \tilde{p}}, \overrightarrow{x_{n_{j}}} \vec{p}\right\rangle
$$




$$
\begin{aligned}
& \leqslant \alpha_{n_{j}}\left\langle\overrightarrow{f\left(x_{n_{j}}\right) \tilde{p}}, \overrightarrow{x_{n_{j}} \vec{p}}\right\rangle+\left(1-\alpha_{n_{j}}\right) d\left(T^{n_{j}} x_{n_{j}}, \tilde{p}\right) d\left(x_{n_{j}}, \tilde{p}\right) \\
& \leqslant \alpha_{n_{j}}\left\langle\overrightarrow{f\left(x_{n_{j}}\right) \tilde{p}}, \overrightarrow{x_{n_{j}} \vec{p}}\right\rangle+\left(1-\alpha_{n_{j}}\right)\left(\left(1+\mu_{n} M\right) d\left(x_{n_{j}}, \tilde{p}\right)+\xi_{n}\right) d\left(x_{n_{j}}, \tilde{p}\right) \\
& =\alpha_{n_{j}}\left\langle\overrightarrow{f\left(x_{n_{j}}\right) \tilde{p}}, \overrightarrow{x_{n_{j}} \vec{p}}\right\rangle+\left(1-\alpha_{n_{j}}\right)\left(\left(1+\mu_{n} M\right) d^{2}\left(x_{n_{j}}, \tilde{p}\right)+\xi_{n} d\left(x_{n_{j}}, \tilde{p}\right)\right) .
\end{aligned}
$$

Since $\sum_{n=1}^{\infty}, \mu_{n}<\infty$ and $\sum_{n=1}^{\infty} \xi_{n}<\infty$, which implies that

$$
\begin{aligned}
d^{2}\left(x_{n_{j}}, \tilde{p}\right) \leqslant\left\langle\overrightarrow{f\left(x_{n_{j}}\right) \tilde{p}}, \overrightarrow{x_{n_{j}} \tilde{p}}\right\rangle & =\left\langle\overrightarrow{f\left(x_{n_{j}}\right) f(\tilde{p})}, \overrightarrow{x_{n_{j}} \vec{p}}\right\rangle+\left\langle\overrightarrow{f(\tilde{p}) \tilde{p}}, \overrightarrow{x_{n_{j}} \vec{p}}\right\rangle \\
& \leqslant d\left(f\left(x_{n_{j}}\right), f(\tilde{p})\right) d\left(x_{n_{j}}, \tilde{p}\right)+\left\langle\overrightarrow{f(\tilde{p})}, \overrightarrow{x_{n_{j}} \vec{p}}\right\rangle \\
& \leqslant k d^{2}\left(x_{n_{j}}, \tilde{p}\right)+\left\langle\overrightarrow{f(\tilde{p}), \tilde{p}}, \overrightarrow{x_{n_{j}} \tilde{p}}\right\rangle,
\end{aligned}
$$

hence,

$$
d^{2}\left(x_{n_{j}}, \tilde{p}\right) \leqslant \frac{1}{1-k}\left\langle\overrightarrow{f(\tilde{p}), \tilde{p}}, \overrightarrow{x_{n_{j}} \vec{p}}\right\rangle
$$

Since $\left\{x_{n_{j}}\right\} \Delta$-converges to $\tilde{p}$, by Lemma 2.6, we have

$$
\limsup _{n \rightarrow \infty}\left\langle\overrightarrow{f(\tilde{p}) \tilde{p}}, \overrightarrow{x_{n_{j}} \tilde{p}}\right\rangle \leqslant 0,
$$

thus,

$$
\limsup _{n \rightarrow \infty}\left\langle\overrightarrow{f(\tilde{p}) \tilde{p}}, \overrightarrow{x_{n} \tilde{p}}\right\rangle \leqslant 0
$$

Step III. Finally, we prove that $x_{n} \rightarrow \tilde{p}$ as $n \rightarrow \infty$ for all $n \in \mathbb{N}$, such that $y_{n}=\beta_{n} x_{n} \oplus\left(1-\beta_{n}\right) T^{n} x_{n}$. Now we set $w_{n}=\alpha_{n} \tilde{p} \oplus\left(1-\alpha_{n}\right) T^{n} y_{n}$. It follows form Lemmas 2.5, 2.7, and 2.8 (i), (ii) that

$$
\begin{aligned}
& d^{2}\left(x_{n+1}, \tilde{p}\right)=d^{2}\left(\alpha_{n} f\left(x_{n}\right) \oplus\left(1-\alpha_{n}\right) T^{n} y_{n}, \tilde{p}\right) \\
& \leqslant d^{2}\left(w_{n}, \tilde{p}\right)+2\left\langle\overrightarrow{x_{n+1} w_{n}}, \overrightarrow{x_{n+1} \vec{p}}\right\rangle \\
& \leqslant\left(\alpha_{n} d(\tilde{p}, \tilde{p})+\left(1-\alpha_{n}\right) d\left(T^{n} x_{n}, \tilde{p}\right)\right)^{2}+2\left[\alpha_{n}\left\langle\overrightarrow{f\left(x_{n}\right) w_{n}}, \overrightarrow{x_{n+1} \tilde{p}}\right\rangle+\left(1-\alpha_{n}\right)\left\langle\overrightarrow{T^{n} y_{n} w_{n}}, \overrightarrow{x_{n+1}} \vec{p}\right\rangle\right] \\
& \leqslant\left(1-\alpha_{n}\right)^{2}\left(\left(1+\mu_{n} M\right) d\left(x_{n}, \tilde{p}\right)+\xi\right)^{2}+2\left[\alpha_{n}^{2}\left\langle\overrightarrow{f\left(x_{n}\right) \tilde{p}}, \overrightarrow{x_{n+1}} \vec{p}\right\rangle\right. \\
& +\alpha_{n}\left(1-\alpha_{n}\right)\left\langle\overrightarrow{f\left(x_{n}\right) T^{n} x_{n}}, \overrightarrow{x_{n+1} \vec{p}}\right\rangle+\alpha_{n}\left(1-\alpha_{n}\right)\left\langle\overrightarrow{T^{n} y_{n} \tilde{p}}, \overrightarrow{x_{n+1} \tilde{p}}\right\rangle \\
& \left.+\left(1-\alpha_{n}\right)^{2}\left\langle\overrightarrow{\mathrm{T}^{n} y_{n} T^{n} x_{n}}, \overrightarrow{x_{n+1}} \vec{p}\right\rangle\right] \\
& \leqslant\left(1-\alpha_{n}\right)^{2}\left(\left(1+\mu_{n} M\right) d\left(x_{n} \tilde{p}\right)+\xi_{n}\right)^{2}+2\left[\alpha_{n}^{2}\left\langle\overrightarrow{f\left(x_{n}\right) \tilde{p}}, \overrightarrow{x_{n+1}} \vec{p}\right\rangle\right. \\
& +\alpha_{n}\left(1-\alpha_{n}\right)\left\langle\overrightarrow{f\left(x_{n}\right) T^{n} x_{n}}, \overrightarrow{x_{n+1} \vec{p}}\right\rangle+\alpha_{n}\left(1-\alpha_{n}\right) d\left(T^{n} y_{n}, \tilde{p}\right) d\left(x_{n+1}, \tilde{p}\right) \\
& \left.+\left(1-\alpha_{n}\right)^{2} d\left(T^{n} y_{n}, T^{n} x_{n}\right) d\left(x_{n+1}, \tilde{p}\right)\right] \\
& \leqslant\left(1-\alpha_{n}\right)^{2}\left(\left(1+\mu_{n} M\right) d\left(x_{n}, \tilde{p}\right)+\xi_{n}\right)^{2}+2\left[\alpha _ { n } ^ { 2 } \left\langle\overrightarrow{f\left(x_{n}\right) \tilde{p}}, \overrightarrow{x_{n+1} \tilde{p}}\right.\right. \\
& +\alpha_{n}\left(1-\alpha_{n}\right)\left\langle\overrightarrow{f\left(x_{n}\right) T^{n} x_{n}}, \overrightarrow{x_{n+1} \tilde{p}}\right\rangle+\alpha_{n}\left(1-\alpha_{n}\right)\left(\left(1+\mu_{n} M\right) d\left(y_{n}, \tilde{p}\right)+\xi_{n}\right) d\left(x_{n+1}, \tilde{p}\right) \\
& \left.+\left(1-\alpha_{n}\right)^{2}\left(\left(1+\mu_{n} M\right) d\left(y_{n}, x_{n}\right)+\xi_{n}\right) d\left(x_{n+1}, \tilde{p}\right)\right] \\
& =\left(1-\alpha_{n}\right)^{2}\left(\left(1+\mu_{n} M\right) d\left(x_{n}, \tilde{p}\right)+\xi_{n}\right)^{2}+2\left[\alpha_{n}^{2}\left\langle\overrightarrow{f\left(x_{n}\right) \vec{p}}, \overrightarrow{x_{n+1}} \vec{p}\right\rangle\right. \\
& +\alpha_{n}\left(1-\alpha_{n}\right)\left(\left(1+\mu_{n} M\right) d\left(y_{n}, \tilde{p}\right)+\xi_{n}\right) d\left(x_{n+1}, \tilde{p}\right) \\
& \left.+\left(1-\alpha_{n}\right)^{2}\left(\left(1+\mu_{n} M\right) d\left(y_{n}, x_{n}\right)+\xi_{n}\right) d\left(x_{n+1}, \tilde{p}\right)\right] \\
& \leqslant\left(1-\alpha_{n}\right)^{2}\left(\left(1+\mu_{n} M\right) d\left(x_{n}, \tilde{p}\right)+\xi_{n}\right)^{2}+2\left[\alpha_{n}^{2} d\left(f\left(x_{n}\right), \tilde{p}\right) d\left(x_{n+1}, \tilde{p}\right)\right. \\
& \left.+\alpha_{n}\left(1-\alpha_{n}\right)\left(\left(1+\mu_{n} M\right) d\left(f\left(x_{n}\right), \tilde{p}\right) d\left(x_{n+1}, \tilde{p}\right)+\xi_{n}\right)+(1-\alpha)^{2}\left(\xi_{n}\right) d\left(x_{n+1}, \tilde{p}\right)\right] .
\end{aligned}
$$


Since $\sum_{n=1}^{\infty} \mu_{n}<\infty$ and $\sum_{n=1}^{\infty} \xi_{n}<\infty$, we get

$$
\begin{aligned}
d^{2}\left(x_{n+1}, \tilde{p}\right) & \leqslant\left(1-\alpha_{n}\right)^{2} d^{2}\left(x_{n}, \tilde{p}\right)+2 \alpha_{n} d\left(f\left(x_{n}\right), \tilde{p}\right) d\left(x_{n+1}, \tilde{p}\right) \\
& =\left(1-\alpha_{n}\right)^{2} d^{2}\left(x_{n}, \tilde{p}\right)+2 \alpha_{n} d\left(f\left(x_{n}\right), f(\tilde{p})\right) d\left(x_{n+1}, \tilde{p}\right)+2 \alpha_{n}\left\langle\overrightarrow{f(\tilde{p}) \tilde{p}}, \overrightarrow{x_{n+1} \tilde{p}}\right\rangle \\
& \leqslant\left(1-\alpha_{n}\right)^{2} d^{2}\left(x_{n}, \tilde{p}\right)+2 \alpha_{n} k d\left(x_{n}, \tilde{p}\right) d\left(x_{n+1}, \tilde{p}\right)+2 \alpha_{n}\left\langle\overrightarrow{f(\tilde{p}) \tilde{p}}, \overrightarrow{x_{n+1} \tilde{p}}\right\rangle \\
& \leqslant\left(1-\alpha_{n}\right)^{2} d^{2}\left(x_{n}, \tilde{p}\right)+\alpha_{n} k\left(d^{2}\left(x_{n}, \tilde{p}\right)+d^{2}\left(x_{n+1}, \tilde{p}\right)\right)+2 \alpha_{n}\left\langle\overrightarrow{f(\tilde{p}) \tilde{p}}, \overrightarrow{x_{n+1} \tilde{p}}\right\rangle .
\end{aligned}
$$

Since the sequences $\left\{\alpha_{n}\right\}$ and $\left\{x_{n}\right\}$ are bounded, there is $M^{*}>0$ such that

$$
\frac{1}{1-\alpha_{n} k} d^{2}\left(x_{n}, \tilde{p}\right) \leqslant M^{*}
$$

It follows that

$$
\begin{aligned}
d^{2}\left(x_{n+1}, \tilde{p}\right) & \leqslant \frac{1}{1-\alpha_{n} k}\left(1-\alpha_{n}\right)^{2} d^{2}\left(x_{n}, \tilde{p}\right)+\frac{\alpha_{n} k}{1-\alpha_{n} k} d^{2}\left(x_{n}, \tilde{p}\right)+2 \alpha_{n}\left\langle\overrightarrow{f(\tilde{p}) \tilde{p}}, \overrightarrow{x_{n+1} \vec{p}}\right\rangle \\
& \leqslant \frac{\left(1-2 \alpha_{n}+\alpha_{n} k\right)}{1-\alpha_{n} k} d^{2}\left(x_{n}, \tilde{p}\right)+\frac{2 \alpha_{n}}{1-\alpha_{n} k}\left\langle\overrightarrow{f(\tilde{p}) \tilde{p}}, \overrightarrow{x_{n+1} \tilde{p}}\right\rangle+\alpha_{n}^{2} M^{*} \\
& \leqslant\left(1-\frac{2 \alpha_{n}(1-k)}{1-\alpha_{n} k}\right) d^{2}\left(x_{n}, \tilde{p}\right)+\alpha_{n}\left(\frac{2}{1-\alpha_{n} k}\left\langle\overrightarrow{f(\tilde{p}) \tilde{p}}, \overrightarrow{x_{n+1} \vec{p}}\right\rangle+\alpha_{n} M^{*}\right) .
\end{aligned}
$$

Now, taking $\zeta_{n}=\frac{2 \alpha_{n}(1-k)}{1-\alpha_{n} k}, \quad \delta_{n}=\alpha_{n}\left(\frac{2}{1-\alpha_{n} k}\left\langle\overrightarrow{f(\tilde{p}) \tilde{p}}, \overrightarrow{x_{n+1} \tilde{p}}\right\rangle+\alpha_{n} M^{*}\right)$. By Lemma 2.9 and (3.1), therefore we conclude that $x_{n} \rightarrow \tilde{p}$ which is a fixed point of $T$ such that $\tilde{p} \in P_{F(T)} f(\tilde{p})$ and also equivalent to the following solution of the variational inequality (1.2). This completes the proof.

Remark 3.2. Since every Hilbert space is a complete CAT(0) space, Theorem 3.1 improves and generalizes the main results in Wangkeeree et al. [34] and $\mathrm{Xu}$ et al. [36].

The following result can be obtained from Theorem 3.1 immediately.

Theorem 3.3. Let $\mathrm{C}$ be a nonempty closed and convex subset of a real Hilbert space $\mathrm{H}$ and $\mathrm{T}: \mathrm{C} \rightarrow \mathrm{C}$ be a total asymptotically nonexpansive mapping with $\sum_{n=1}^{\infty} \mu_{n}<\infty$ such that $\mathrm{F}(\mathrm{T}) \neq \emptyset$. Let $\mathrm{f}$ be a contraction on $\mathrm{C}$ with the coefficient $k \in[0,1)$ and for arbitrary initial point $x_{1} \in \mathrm{C}$. Let sequence $\left\{x_{n}\right\}$ be the generated by

$$
\left\{\begin{array}{l}
x_{n+1}=\alpha_{n} f\left(x_{n}\right)+\left(1-\alpha_{n}\right) T^{n} y_{n} \\
y_{n}=\beta_{n} x_{n}+\left(1-\beta_{n}\right) T^{n} x_{n}
\end{array}\right.
$$

for all $n \geqslant 1$, where $\alpha_{n}, \beta_{n} \in[0,1]$, which satisfies the conditions (i)-(iv) as in Theorem 3.1. Then the sequence $\left\{x_{n}\right\}$ converges strongly to $\tilde{p}$ such that

$$
\tilde{p}=P_{F(T)} f(\tilde{p}),
$$

which is also equivalent to the solution of the variational inequality

$$
\langle\tilde{p}-f(\tilde{p}), p-\tilde{p}\rangle \geqslant 0, \quad \forall p \in F(T) .
$$

\subsection{Consequently results}

From Theorem 3.1 if we set $\mu_{n}:=k_{n}-1, \xi_{n}:=0, \forall n \geqslant 1$, and $\psi(t):=t, t \geqslant 0$, then we obtain the following result immediately.

Corollary 3.4. Let $\mathrm{C}$ be a nonempty closed convex subset of a complete $\mathrm{CAT}(0)$ space $\mathrm{X}$ and $\mathrm{T}: \mathrm{C} \rightarrow \mathrm{C}$ be an asymptotically nonexpansive mapping with a sequence $\left\{k_{n}\right\} \subset[1, \infty)$ and $\lim _{n \rightarrow \infty} k_{n}=1$ such that $\mathrm{F}(\mathrm{T}) \neq \emptyset$. Let $\mathrm{f}: \mathrm{C} \rightarrow \mathrm{C}$ be a contraction with coefficient $\mathrm{k} \in[0,1)$ and, for arbitrary initial point $\mathrm{x}_{1} \in \mathrm{C}$. Let $\left\{\mathrm{x}_{\mathrm{n}}\right\}$ be generated by (1.4) satisfying the following conditions: 
(i) $\lim _{n \rightarrow \infty} \alpha_{n}=0$;

(ii) $\sum_{n=0}^{\infty} \alpha_{n}=\infty$;

(iii) $\lim _{n \rightarrow \infty} \frac{k_{n}-1}{\alpha_{n}}=0$;

(iv) $\frac{\left|\alpha_{n}-\alpha_{n-1}\right|}{\alpha_{n}^{2}} \rightarrow 0$, as $\mathrm{n} \rightarrow \infty$;

(v) T satisfies the asymptotically regular $\lim _{n \rightarrow \infty} \mathrm{d}\left(\mathrm{x}_{\mathrm{n}}, \mathrm{T}^{\mathrm{n}} \mathrm{x}_{\mathrm{n}}\right)=0$.

Then $\left\{x_{n}\right\}$ converges strongly to $\tilde{p}=P_{F(T)} f(\tilde{p})$, which is a fixed point of $T$ such that $\tilde{p} \in P_{F(T)} f(\tilde{p})$ and also equivalent to the solution of the variational inequality (1.2).

From Theorem 3.1, if $\mu_{n}=0, \xi_{n}=0$, and $n=1$, then we obtain the following result immediately.

Corollary 3.5. Let $\mathrm{C}$ be a nonempty closed and convex subset of complete $\mathrm{CAT}(0)$ space $\mathrm{X}$ and $\mathrm{T}: \mathrm{C} \rightarrow \mathrm{C}$ be a nonexpansive mapping with $\mathrm{F}(\mathrm{T}) \neq \emptyset$. Let $\mathrm{f}: \mathrm{C} \rightarrow \mathrm{C}$ be a contraction with coefficient $\mathrm{k} \in[0,1)$ and, for arbitrary initial point $x_{1} \in C$. Let $\left\{x_{n}\right\}$ be generated by (1.4) and satisfies the following conditions:

(i) $\lim _{n \rightarrow \infty} \alpha_{n}=0$;

(ii) $\sum_{n=0}^{\infty} \alpha_{n}=\infty$;

(iii) $\frac{\left|\alpha_{n}-\alpha_{n-1}\right|}{\alpha_{n}^{2}} \rightarrow 0$ as $n \rightarrow \infty$.

Then $\left\{x_{n}\right\}$ converges strongly to $\tilde{p}$ such that $\tilde{p}=P_{F(T)} f(\tilde{p})$, which is a fixed point of $T$ such that $\tilde{p} \in \mathrm{P}_{\mathrm{F}(\mathrm{T})} \mathrm{f}(\tilde{\mathrm{p}})$ and also equivalent to the solution of the variational inequality (1.2).

Since every Hilbert space is a complete CAT(0) space, we obtain results form Corollary 3.4 and 3.5 respectively.

Corollary 3.6. Let $\mathrm{C}$ be nonempty closed and convex subset of a real Hilbert space $\mathrm{H}$ and $\mathrm{T}: \mathrm{C} \rightarrow \mathrm{C}$ be an asymptotically nonexpansive mapping with a sequence $\left\{k_{n}\right\} \subset[1, \infty)$ and $\lim _{n \rightarrow \infty} k_{n}=1$ such that $\mathrm{F}(\mathrm{T}) \neq \emptyset$. Let $\mathrm{f}: \mathrm{C} \rightarrow \mathrm{C}$ be a contraction with coefficient $\mathrm{k} \in[0,1)$ and, for arbitrary initial point $\mathrm{x}_{1} \in \mathrm{C}$. Let $\left\{\mathrm{x}_{\mathrm{n}}\right\}$ be generated by (3.2) satisfying the conditions (i)-(v) as in Corollary 3.4. Then $\left\{x_{n}\right\}$ generated by (3.2) converges strongly to $\tilde{p}$, which is also equivalent to the solution of the variational inequality (3.3).

Corollary 3.7. Let $\mathrm{C}$ be nonempty closed and convex subset of a real Hilbert space $\mathrm{H}$ and $\mathrm{T}: \mathrm{C} \rightarrow \mathrm{C}$ be a nonexpansive mapping with $\mathrm{F}(\mathrm{T}) \neq \emptyset$. Let $\mathrm{f}: \mathrm{C} \rightarrow \mathrm{C}$ be a contraction with coefficient $\mathrm{k} \in[0,1)$ and, for arbitrary initial point $\mathrm{x}_{1} \in \mathrm{C}$. Let $\left\{\mathrm{x}_{\mathrm{n}}\right\}$ be generated by (3.2) satisfying the conditions (i)-(iii) as in Corollary 3.5. Then $\left\{\mathrm{x}_{\mathrm{n}}\right\}$ converges strongly to $\tilde{p}$, which is also equivalent to the solution of the variational inequality (3.3).

\subsection{An example of numerical results}

In this section, we will illustrate reckoning the convergence behavior of modified two-step viscosity iteration process (1.4) for total asymptotically nonexpansive mappings with the reckoning numerical results for supporting the main theorem.

Example 3.8. Let $X=\mathbb{R}$ be a Euclidean metric space, which is also a complete $\mathrm{CAT}(0)$ space and $\mathrm{C}=$ $[1,10]$. Let $\mathrm{T}: \mathrm{C} \rightarrow \mathrm{C}$ be defined by

$$
\mathrm{T} x=\sqrt[3]{3 x^{2}-2 x+6}
$$

It's obvious that $T$ is a total asymptotically nonexpansive mapping. Let $f: C \rightarrow C$ be defined by

$$
f x=\frac{x+3}{2} .
$$

It's easy to check that $f$ is a contraction mapping. 
Table 1: The values of sequence $\left\{x_{n}\right\}$ and the error values.

\begin{tabular}{|c|c|c|}
\hline number of iterates & $\chi_{n}$ & $\left|x_{n}-p\right|$ \\
\hline 1 & 10.00000000000 & 7.000000000000 \\
2 & 6.785274779613 & 3.785274779613 \\
4 & 3.839539421270 & 0.839539421270 \\
5 & 3.092398253180 & 0.092398253180 \\
6 & 3.006483269122 & 0.006483269122 \\
7 & 3.000356881905 & 0.000356881905 \\
8 & 3.000017184442 & 0.000017184442 \\
9 & 3.000000757059 & $7.5705927 \mathrm{E}-07$ \\
10 & 3.000000031042 & $3.1041818 \mathrm{E}-08$ \\
11 & 3.000000001193 & $1.1927735 \mathrm{E}-09$ \\
12 & 3.000000000043 & $4.3094417 \mathrm{E}-11$ \\
13 & 3.000000000001 & $1.4672707 \mathrm{E}-12$ \\
14 & 3.000000000000 & $4.7073456 \mathrm{E}-14$ \\
15 & 3.000000000000 & $1.3322676 \mathrm{E}-15$ \\
& 3.000000000000 & 0.00000000000 \\
\hline
\end{tabular}

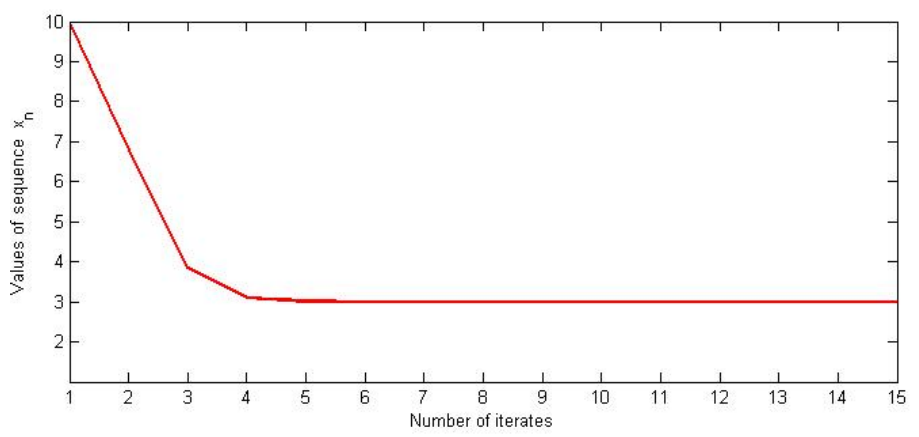

Figure 1: Convergence behavior of the sequence $\left\{x_{n}\right\}$ in Table 1.

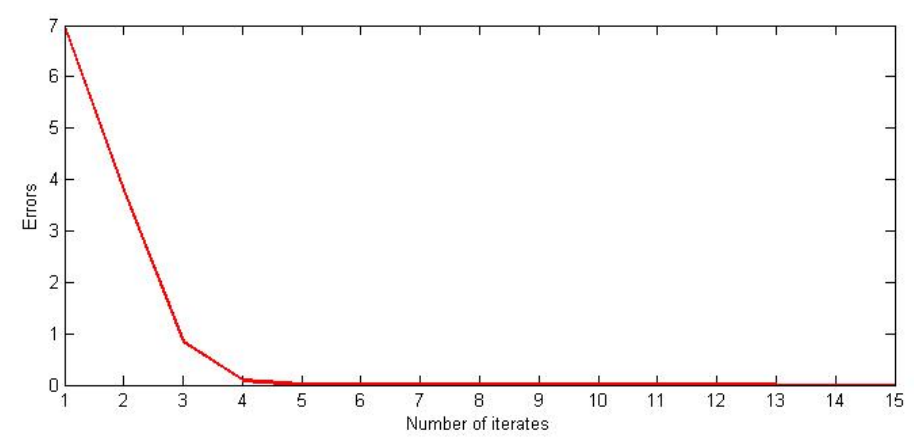

Figure 2: Convergence behavior of the errors in Table 1.

Setting $\alpha_{n}=\frac{n^{2}-2 n+4}{n^{3}+18 n-15}$ and $\beta_{n}=\frac{e^{\frac{1}{n}}-1}{n}$ for all $n \geqslant 1$ and starting point $x_{1}=10$, then we obtain numerical results in Table 1 and we see that $F(T)=\{3\}$. Furthermore, we then illustrate the convergence behavior of modified two-step viscosity iteration process (1.4) by the values of sequence $\left\{x_{n}\right\}$ and errors are shown in Figures 1 and 2, respectively. 
Next, we will show that a fixed point of mapping $\mathrm{T}$ is solution of the variational inequality

$$
\langle\overrightarrow{\tilde{p} f(\tilde{p})}, \vec{p} \overrightarrow{\tilde{p}}\rangle \geqslant 0, \quad \forall p \in F(T),
$$

where $\tilde{p}=P_{F(T)} f(\tilde{p})$.

Proof. Let $p \in F(T)$, we have $F(T)=\{3\}$, thus $p=3$. By Theorem 3.1, we have

$$
p=\tilde{p}=P_{F(T)} f(\tilde{p}) .
$$

From variational inequality that

$$
\langle\overrightarrow{\tilde{p} f(\tilde{p})}, \overrightarrow{p \mathfrak{p}}\rangle \geqslant 0, \quad\langle(3) \cdot f(3),(3) \cdot(3)\rangle \geqslant 0, \quad\langle(3) \cdot(3),(3) \cdot(3)\rangle \geqslant 0
$$

Therefore a fixed point of mapping $\mathrm{T}$ which is a solution satisfying the variational inequality.

\section{Some applications}

Now, we give some applications (see [40] and reference cited therein) of consequently results. In particular, Corollary 3.7.

\subsection{Applications to a nonlinear Volterra integral equation}

Let us consider the following nonlinear Volterra integral equation type

$$
x(t)=g(t)+\int_{0}^{t} F(t, s, x(s)) d s
$$

for all $t \in[0,1]$, where $g$ is a continuous function on $[0,1]$ and $F:[0,1] \times[0,1] \times \mathbb{R} \rightarrow \mathbb{R}$ is continuous and satisfies the following condition

$$
|F(t, s, x)-F(t, s, y)| \leqslant|x-y|
$$

for all $t, s \in[0,1]$ and $x, y \in \mathbb{R}$. Define a mapping $T: \mathrm{L}^{2}[0,1] \rightarrow \mathrm{L}^{2}[0,1]$ by

$$
(T x)(t)=g(t)+\int_{0}^{t} F(t, s, x(s)) d s
$$

for all $t \in[0,1]$. It is obvious to see that $T$ is a nonexpansive mapping. This means that finding a solution of the integral equation (4.5) is reduced to finding a fixed point of the nonexpansive mapping $\operatorname{T}_{\text {in }} \mathrm{L}^{2}[0,1]$. For any given function $x_{0} \in \mathrm{L}^{2}[0,1]$, generate sequence $\left\{x_{n}\right\}$ of functions in $\mathrm{L}^{2}[0,1]$ by

$$
\left\{\begin{array}{l}
x_{n+1}=\alpha_{n} f\left(x_{n}\right)+\left(1-\alpha_{n}\right) T y_{n} \\
y_{n}=\beta_{n} x_{n}+\left(1-\beta_{n}\right) T x_{n}, \forall n \geqslant 0
\end{array}\right.
$$

The following result will be obtained from Corollary 3.7 immediately.

Theorem 4.1. Let $\mathrm{F}, \mathrm{g}, \mathrm{T}, \mathrm{L}^{2}[0,1]$ be the same as above. Let $\mathrm{f}$ be a contraction on $\mathrm{L}^{2}[0,1]$ with the coefficient $k \in[0,1)$. Let $\left\{x_{n}\right\}$ be generated by (4.2). If $\mathrm{F}(\mathrm{T}) \neq \emptyset$ and $\alpha_{n}, \beta_{n} \in(0,1)$ satisfy the conditions (i)-(iv) in Corollary 3.5, then $\left\{x_{n}\right\}$ converges strongly in $\mathrm{L}^{2}[0,1]$ to a solution of the integral equation (4.1), its is also a solution of the variational inequality:

$$
\langle\tilde{\mathrm{q}}-\mathrm{f}(\tilde{\mathrm{q}}), \mathrm{q}-\tilde{\mathrm{q}}\rangle \geqslant 0 ; \quad \forall \mathrm{q} \in \mathrm{F}(\mathrm{T}) .
$$

\subsection{Application to variational inequality}

Consider the variational inequality (in short term, VI)

$$
\langle A \tilde{x}, x-\tilde{x}\rangle \geqslant 0, x \in C,
$$

where $A$ is a monotone operator (single-valued) in Hilbert space $\mathrm{H}$ and $\mathrm{C}$ is a closed convex subset of $\mathrm{H}$ with $C \subset \operatorname{dom}(A)$. 
The example of (4.3) is the constrained minimization problem

$$
\min _{x \in C} \varphi(x)
$$

where $\varphi: H \rightarrow \mathbb{R}$ is a proper convex and lower-semicontinuous function. If $\varphi$ is (Fréchet) differentiable, then the minimization problem (4.4) is equivalently reformulated as (4.3) with $A=\nabla \varphi$. Notice that the (VI) (4.3) is equivalent to the fixed point problem

$$
\mathrm{T} \tilde{x}=\tilde{x}, \quad T x=P_{C}(I-\lambda A) x,
$$

for all $\lambda>0$. If $A$ is Lipschitzian and strongly monotone, then for $\lambda>0$ small enough, $T$ is a contraction and its unique fixed point is also the unique solution of the VI (4.3). Whenever, if $A$ is not strongly monotone, $\mathrm{T}$ is no longer a contraction, in general. In this case we must deal with nonexpansive mappings for solving the VI (4.3). More precisely, we assume

(i) $\mathrm{A}$ is L-Lipschitzian if there exists $\mathrm{L}>0$, such that,

$$
\|A x-A y\| \leqslant L\|x-y\|, x, y \in H .
$$

(ii) $A$ is $\sigma$-inverse strongly monotone $(\sigma$-ism) if there exists $\sigma>0$, that is,

$$
\langle A x-A y, x-y\rangle \geqslant \sigma\|A x-A y\|^{2}, x, y \in H .
$$

Note that if $\nabla \varphi$ is L-Lipschitzian, then $\nabla \varphi$ is $\frac{1}{\mathrm{~L}}$-ism.

Under the conditions (i) and (ii), it is well known [6] that the operator $T=P_{C}(I-\lambda A)$ is nonexpansive provided that $0<\lambda<2 \sigma$.

The following result will be obtained from Corollary 3.7 immediately.

Theorem 4.2. Suppose that the VI (4.3) is solvable. Assume also A satisfies (i) and (ii), and $0<\lambda<2 \sigma$. Let $\mathrm{f}: \mathrm{C} \rightarrow \mathrm{C}$ be a contraction. Then sequence $\left\{\mathrm{x}_{\mathrm{n}}\right\}$ is generated by

$$
\left\{\begin{array}{l}
x_{n+1}=\alpha_{n} f\left(x_{n}\right)+\left(1-\alpha_{n}\right) P_{C}(I-\lambda A) y_{n} \\
y_{n}=\beta_{n} x_{n}+\left(1-\beta_{n}\right) P_{C}(I-\lambda A) x_{n}, \forall n \geqslant 0
\end{array}\right.
$$

where $\alpha_{n}, \beta_{n} \in(0,1)$. In addition, assume that the conditions (i)-(iii) in Corollary 3.7 are satisfied. Then $\left\{x_{n}\right\}$ converges in norm to a solution $\tilde{x}$ of the VI (4.3) which is also a solution to the VI

$$
\langle(I-f)(\tilde{x}), x-\tilde{x}\rangle \geqslant 0, \quad x \in A^{-1}(0) .
$$

\subsection{Application to hierarchical minimization}

We next consider a hierarchical minimization problem (see [27]).

Let $\varphi_{0}, \varphi_{1}: H \rightarrow R$ be a lower semicontinuous convex function. Consider the following hierarchical minimization problem:

$$
\min _{x \in S_{0}} \varphi_{1}(x), \quad S_{0}=\arg \min _{x \in H} \varphi_{0}(x) .
$$

Now, we always assume that $S_{0}$ is nonempty. Let $S=\arg \min _{x \in S_{0}} \varphi_{1}(x)$ and assume $S \neq \emptyset$. Suppose that $\varphi_{0}$ and $\varphi_{1}$ are differentiable and their gradients satisfy the Lipschitz continuity conditions:

$$
\left\|\nabla \varphi_{0}(\mathrm{x})-\nabla \varphi_{0}(\mathrm{y})\right\| \leqslant \mathrm{L}_{0}\|\mathrm{x}-\mathrm{y}\|, \quad\left\|\nabla \varphi_{1}(\mathrm{x})-\nabla \varphi_{1}(\mathrm{y})\right\| \leqslant \mathrm{L}_{1}\|\mathrm{x}-\mathrm{y}\| .
$$

Note that the condition (4.7) implies that $\nabla \varphi_{i}$ is $\frac{1}{L_{i}}-i s m(i=0,1)$. Now let

$$
\mathrm{T}_{0}=\mathrm{I}-\gamma_{0} \nabla \varphi_{0}, \quad \mathrm{~T}_{1}=\mathrm{I}-\gamma_{1} \nabla \varphi_{1}
$$


where $\gamma_{0}>0$ and $\gamma_{1}>0$. Note that $T_{i}$ is nonexpansive [6] if $0<\gamma_{i}<\frac{2}{L_{i}}(i=0,1)$. Also, it is obviously seen that $S_{0}=\operatorname{Fix}\left(T_{0}\right)$.

The optimality condition for $\tilde{x} \in S_{0}$ to be a solution of the hierarchical minimization (4.6) is the VI:

$$
\tilde{x} \in \mathrm{S}_{0},\left\langle\nabla \varphi_{1}(\tilde{x}\rangle \geqslant 0, \quad x \in \mathrm{S}_{0} .\right.
$$

This is the VI (4.3) with $C=S_{0}$ and $A=\nabla \varphi_{1}$.

The following result will be obtained from Corollary 3.7 immediately.

Theorem 4.3. Assume the hierarchical minimization problem (4.6) is solvable. Let $\mathrm{f}: \mathrm{C} \rightarrow \mathrm{C}$ be a contraction. Then sequence $\left\{x_{n}\right\}$ is generated by

$$
\left\{\begin{array}{l}
x_{n+1}=\alpha_{n} f\left(x_{n}\right)+\left(1-\alpha_{n}\right) P_{S_{0}}\left(I-\lambda \nabla \varphi_{1}\right) y_{n}, \\
y_{n}=\beta_{n} x_{n}+\left(1-\beta_{n}\right) P_{S_{0}}\left(I-\lambda \nabla \varphi_{1}\right) x_{n}, \forall n \geqslant 0
\end{array}\right.
$$

where $\alpha_{n}, \beta_{n} \in(0,1)$. In addition, assume that the conditions (i)-(iii) in Corollary 3.7 are satisfied. If the condition (4.7) is satisfied and $0<\gamma_{i}<\frac{2}{\mathrm{~L}_{i}}(i=0,1)$, then $\left\{x_{n}\right\}$ convergence in norm to a solution $\tilde{x}$ of the VI (4.8) that is, a solution of hierarchical minimization problem (4.6) which also solves the VI

$$
\langle(I-f)(\tilde{x}), x-\tilde{x}\rangle \geqslant 0, \quad x \in S .
$$

\subsection{Applications to a nonlinear variation inclusion problem}

Let $\mathrm{H}$ be a real Hilbert space and $\mathrm{K}: \mathrm{H} \rightarrow 2^{\mathrm{H}}$ be a multi-valued maximal monotone mapping. Then the resolvent mapping $\mathrm{J}_{\lambda}^{\mathrm{K}}: \mathrm{H} \rightarrow \mathrm{H}$ associated with $\mathrm{K}$ is defined by

$$
\mathrm{J}_{\lambda}^{\mathrm{K}}(\mathrm{x}):=(\mathrm{I}+\lambda \mathrm{K})^{-1}(\mathrm{x})
$$

for all $x \in H$ and for some $\lambda>0$, where I stands for the identity mapping on $H$.

We note that, for all $\lambda>0$, the resolvent mapping $J_{\lambda}^{K}$ is a single-valued nonexpansive mapping.

The so-called monotone variational inclusion problem (in short term, MVIP) with respect to $K$ is to find $x^{*} \in \mathrm{H}$ such that

$$
0 \in \mathrm{K}\left(\mathrm{x}^{*}\right) \text {. }
$$

From the definition of the resolvent mapping $J_{\lambda}^{K}$, it is easy to know that the problem MVIP (4.9) is equivalent to find $x^{*} \in \mathrm{H}$ such that

$$
x^{*} \in \operatorname{Fix}\left(\mathrm{J}_{\lambda}^{\mathrm{K}}\right)
$$

for some $\lambda>0$. For any given function $x_{0} \in H$, generated sequence $\left\{x_{n}\right\}$ by

$$
\left\{\begin{array}{l}
x_{n+1}=\alpha_{n} f\left(x_{n}\right)+\left(1-\alpha_{n}\right) J_{\lambda}^{K} y_{n} \\
y_{n}=\beta_{n} x_{n}+\left(1-\beta_{n}\right) J_{\lambda}^{K} x_{n}, \forall n \geqslant 0
\end{array}\right.
$$

where $f: H \rightarrow H$, is a contraction.

The following result will be obtained from Corollary 3.7 immediately.

Theorem 4.4. Let $\mathrm{K}$ and $\mathrm{J}_{\lambda}^{\mathrm{K}}$ be the same as above. Let $\mathrm{f}: \mathrm{H} \rightarrow \mathrm{H}$ be a contraction and $\left\{\mathrm{x}_{\mathrm{n}}\right\}$ be generated by (4.10). If $\alpha_{n}, \beta_{n} \in(0,1)$ satisfy the conditions (i)-(iii) as in Corollary 3.7 and $F\left(J_{\lambda}^{K}\right) \neq \emptyset$, then $\left\{x_{n}\right\}$ converges strongly to a solution of MVIP (4.9), which is a solution of the variational inequality:

$$
\langle\tilde{p}-f(\tilde{q}), q-\tilde{q}\rangle \geqslant 0, \quad \forall q \in F\left(J_{\lambda}^{K}\right) .
$$

\section{Acknowledgment}

The first author was financial supported by the Rajamangala University of Technology Thanyaburi (RMUTT). The second author would like to thank the Research Professional Development Project Under the Science Achievement Scholarship of Thailand (SAST) for financial support. 


\section{References}

[1] B. Ahmadi Kakavandi, Weak topologies in complete CAT(0) Metric spaces, Proc. Am. Math. Soc., 141 (2013), 10291039. 2, 2.6

[2] B. Ahmadi Kakavandi, M. Amini, Duality and subdifferential for convex function on complete CAT(0) metric spaces, Nonlinear Anal., 73 (2010), 3450-3455. 2, 2

[3] N. Akkasriworn, D. Kitkuan, A. Padcharoen, Convergence theorems for generalized I-asymptotically nonexpansive mappings in a Hadamard spaces, Commun. Korean Math. Soc. 31 (2016), 483-493. 1

[4] Y. I. Alber, C. E. Chidume, H. Zegeye, Approximating fixed points of total asymptotically nonexpansive mappings, Fixed Point Theory Appl., 2006 (2006), 20 pages. 1

[5] M. Başarir, A. Şahin, On the strong and $\Delta$-convergence for total asymptotically nonexpansive mappings on a CAT(0) space, Carpathian Math. Publ., 5 (2013), 170-179. 1

[6] I. D. Berg, I. G. Nikolaev, Quasilinearization and curvature of Aleksandrov spaces, Geom. Dedicata, 133 (2008), $195-218$. $1,4.2,4.3$

[7] M. R. Bridson, A. Haefliger, Metric spaces of Non-positive Curvature, Springer-Verlag, Berlin, (1999). 1

[8] K. S. Brown, Buildings, Springer, New York, (1998). 1

[9] S. S. Chang, L. Wang, H. W. J. Lee, C. K. Chan, Strong and $\Delta$-convergence for mixed type total asymptotically nonexpansive mappings in CAT(0) spaces, Fixed Point Theory Appl., 2013 (2013), 16 pages. 1

[10] S. S. Chang, L. Wang, H. W. J. Lee, C. K. Chan, L. Yang, Demiclosed principle and $\Delta$-convergence theorems for total asymptotically nonexpansive mapping in CAT(0) space, Appl. Math. Comput., 219 (2012), 2611-2617. 1

[11] Y. J. Cho, L. Ćirić, S.-H. Wang, Convergence theorems for nonexpansive mappings in CAT(0) spaces, Nonlinear Anal., 74 (2011), 6050-6059. 1

[12] P. Cholamjiak, A. A. N. Abdou, Y. J. Cho, Proximal point algorithms involving fixed points of nonexpansive mappings in CAT(0) spaces, Fixed Point Theory Appl., 2015 (2015), 13 pages. 1

[13] H. Dehghan, J. Rooin, A Characterization of metric projection in CAT(0) spaces, Proc. Inter. Con. Func. Equation, Geo. Func. Appli., 2012 (2012), 3 pages. 1

[14] S. Dhompongsa, W. A. Kirk, B. Panyanak, Nonexpansive set-valued mappings in metric and Banach spaces, J. Nonlinear Convex Anal., 8 (2007), 33-45. 2.4

[15] S. Dhompongsa, W. A. Kirk, B. Sims, Fixed points of uniformly Lipschitzian mappings, Nonlinear Anal., 65 (2006), 762-772. 2

[16] S. Dhompongsa, B. Panyanak, On $\Delta$-convergence theorems in CAT(0) spaces, Comput. Math. Appl., 56 (2008), 25722579. 2.1

[17] K. Goegel, W. A. Kirk, A fixed point theorem for asymptotically nonexpansive mappings, Proc. Am. Math. Soc., 35 (1972), 171-174. 1

[18] E. Karapınar, H. Salahifard, S. M. Vaezpour, Demiclosedness principle for total asymptoticallty nonexpansive mappings in CAT(0) spaces, J. Appl. Math., 2014 (2014), 10 pages. 1, 2.5

[19] W. A. Kirk, B. Panyanak, A concept of convergence in geodesic spaces, Nonlinear Anal., 68 (2008), 3689-3696. 2, 2.3

[20] T. C. Lim, Remarks on some fixed point theorems, Proc. Amer. Math. Soc., 60 (1976), 179-182. 2

[21] N. Pakkaranang, P. Kumam, Strong and $\Delta$-convergence theorems for asymptotically k-Strictly pseudo-contractive mappings in CAT(0) spaces, Commu. Math. Appl., 7 (2016), 189-197. 1

[22] N. Pakkaranang, P. Kumam, Y. J. Cho, Proximal point algorithms for solving convex minimization problem and common fixed points problem of asymptotically quasi-nonexpansive mappings in CAT(0) spaces with convergence analysis, Numer. Algor., 2017 (2017), 19 pages.

[23] N. Pakkaranang, P. Kumam, Y. J. Cho, P. Saipara, A. Padcharoen, C. Khaofong, Strong convergence of modified viscosity implicit approximation methods for asymptotically nonexpansive mappings in complete CAT(0) spaces, J. Math. Computer Sci., 17 (2017), 345-354.

[24] N. Pakkaranang, P. S. Ngiamsunthorn, P. Kumam, Y. J. Cho, Convergence theorems of the modified S-type iterative method for $(\alpha, \beta)$-generalized hybrid mapping in CAT(0) Spaces, J. Math. Anal., 8 (2017), 103-112. 1

[25] D. R. Sahu, Fixed points of demicontinuous nearly Lipschitzian mappings in Banach spaces, Comment. Math. Univ. Carolin., 46 (2005), 653-666. 1

[26] P. Saipara, P. Chaipunya, Y. J. Cho, P. Kumam, On strong and $\Delta$-convergence of modified S-iteration for uniformly continuous total asymptotically nonexpansive mappings in CAT (K) spaces, J. Nonlinear Sci. Appl., 8 (2015), 965-975. 2.2

[27] P. Sunthrayuth, Y. J. Cho, P. Kumam, Vicosity approximation methods for zeros of accretive operator and fixed point problems in Banach spaces, Bull. Malays. Math. Sci. Soc., 39 (2016), 773-793. 4.3

[28] J. F. Tang, S. S. Chang, H. W. J. Lee, C. K. Chan, Iterative algorithm and $\Delta$-convergence theorems for total asymptotically nonexpansive mappings in CAT(0) spaces, Abstr. Appl. Anal., 2012 (2012), 11 pages. 1

[29] I. Uddin, J. Ali, J. J. Nieto, An iteration scheme for a family of multivalued mappings in CAT(0) spaces with an application to image recovery, RACSAM, 2017 (2017), 12 pages. 1

[30] I. Uddin, J. J. Nieto, J. Ali, One-step iteration scheme for multivalued nonexpansive mappings in CAT(0) spaces, Mediterr. J. Math., 13 (2016), 1211-1225. 1 
[31] L.-L. Wan, $\Delta$-Convergence for mixed-type total asymptotically nonexpansive mappings in hyperbolic spaces, J. Inequal. Appl., 2013 (2013), 8 pages. 1

[32] L. Wang, S.-S. Chang, Z. Ma, Convergence theorems for total asymptotically nonexpansive non-self mappings in CAT(0) spaces, J. Inequal. Appl., 2013 (2013), 10 pages. 1

[33] R. Wangkeeree, U. Boonkong, P. Preechasilp, Viscosity approximation methods for asymptotically nonexpansive mapping in CAT(0) spaces, Fixed point Theory Appl., 2015 (2015), 15 pages. 1, 1

[34] R. Wangkeeree, P. Preechasilp, Viscosity approximation methods for nonexpansive mappings in CAT(0) spaces, J. Inequal. Appl., 2013 (2013), 15 pages. 1, 2, 2.7, 2.8, 3.2

[35] H.-K. Xu, An iterative approach to quadratic optimization, J. Optim. Theory Appl., 116 (2003), 659-678. 2.9

[36] H.-K. Xu, M. A. Alghamdi, N. Shahzad, The viscosity technique for the implicit midpoint rule of nonexpansive mappings in Hilbert spaces, Fixed Point Theory Appl., 2015 (2015), 12 pages. 3.2

[37] L. Yang, F. H. Zhao, Strong and $\Delta$-convergence theorems for total asymptotically nonexpansive non-self mappings in $C A T(\mathrm{~K})$ spaces, J. Inequal. Appl., 2013 (2013), 17 pages. 1

[38] L.-C. Zhao, S.-S. Chang, J. K. Kim, Mixed type iteration for total asymptotically nonexpansive mappings mappings in Hyperbolic spaces, Fixed point Theory Appl., 2013 (2013), 11 pages.

[39] L.-C. Zhao, S.-S. Chang, X. R. Wang, Convergence theorems for total asymptotically nonexpansive mappings in Hyperbolic spaces, J. Appl. Math., 2013 (2013), 5 pages. 1

[40] L.-C. Zhao, S.-S. Chang, C.-F. Wen, Viscosity approximation mathods for the implicit midpoint rule of asymptotically nonexpansive mappings in Hulbert spaces, J. Nonlinear Sci. Appl., 9 (2016), 4478-4488. 4 